\title{
A systematic review of behavioural weight-loss interventions involving primary-care physicians in overweight and obese primary-care patients (1999-2011)
}

\author{
Sze Lin Yoong*, Mariko Carey, Rob Sanson-Fisher and Alice Grady \\ Priority Research Centre for Health Behaviour, School of Medicine and Public Health, University of Newcastle, \\ Callaghan, NSW 2308, Australia and Hunter Medical Research Institute, Newcastle, NSW, Australia
}

Submitted 13 June 2012: Final revision received 23 August 2012: Accepted 24 August 2012: First published online 260 0ctober 2012

\begin{abstract}
Objective: The present review aimed to examine the effectiveness of behavioural weight-loss interventions involving primary-care physicians in producing weight loss in overweight and obese primary-care patients.

Design: A systematic review was conducted by searching online databases (MEDLINE, EMBASE, Cochrane, PsycINFO and SCOPUS) from January 1999 to December 2011. All abstracts were screened and coded for eligibility. The Cochrane Effective Practice and Organisation of Care Group quality criteria were used to assess the methodological adequacy of included studies. Information related to study design, population characteristics and intervention details was extracted.

Setting: Primary care.

Subjects: Overweight or obese (defined as having a BMI $\geq 25 \cdot 0 \mathrm{~kg} / \mathrm{m}^{2}$ ) primarycare patients.

Results: Sixteen different studies were included. Of these, six assessed primarycare physicians' delivery of weight-loss counselling; nine assessed weight-loss counselling delivered by non-physician personnel with monitoring by primarycare physicians; and one assessed a multi-component intervention. Overall, highintensity weight-loss counselling by primary-care physicians resulted in moderate but not clinically significant weight loss. High-intensity weight-loss counselling delivered by non-physicians, meal replacements delivered in conjunction with dietitian counselling and referral to commercial weight-loss centre programmes accompanied by regular monitoring by a primary-care physician were effective in producing clinically significant weight loss. Dietitian-delivered care appeared effective in producing weight loss regardless of level of intervention intensity. Conclusions: Overall, there were few studies on this topic and the methodological rigour of some included studies was poor. Additional studies assessing the effectiveness and acceptability of potential interventions are needed to confirm these findings.
\end{abstract}

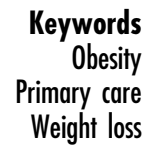

Keywords Weight loss
Obesity is one of the largest modifiable threats to public health in developed countries ${ }^{(1)}$. It affects a large proportion of the population in developed countries and is associated with chronic diseases such as CVD, type 2 diabetes and some cancers ${ }^{(2)}$. The rates of overweight and obesity have been steadily increasing in countries such as the USA, Australia and the $\mathrm{UK}^{(3)}$. A modest weight loss of $5 \%$ in those obese has been shown to be beneficial in improving blood sugar control, CVD-related biomarkers and overall quality of life $\mathrm{e}^{(4)}$.
Primary-care physicians provide first-line health care in many countries. In Australia, more than $80 \%$ of the population consult their primary-care physician at least once per annum $^{(5)}$. The average primary-care physician consultation rate in the UK rose from 3.9 consultations per person in 1995 to $5 \cdot 3$ in $2006^{(6)}$. While more women and older people present for care ${ }^{(5)}$, primary-care physicians still have access to a large proportion of the general population. Both patients $^{(7)}$ and physicians ${ }^{(8)}$ perceive weight management to be part of a primary-care physician's role. Primary-care 
physicians have reported being interested in helping patients manage their weight, but face practical constraints in doing so ${ }^{(9)}$. Those who have been advised by their primary-care physician to lose weight are more likely to try to do so ${ }^{(10)}$. Primary-care physicians are also likely to have multiple opportunities to identify excess weight and deliver ongoing weight-management care required for sustained weight loss.

Despite the advantages of using primary care for interventions targeting obesity, the effectiveness of interventions in this setting has not been widely evaluated. Previous systematic reviews have identified bariatric surgery $^{(11)}$ and pharmacological treatments ${ }^{(12)}$ as potentially effective methods for weight reduction; however, these interventions are costly and are usually indicated for the morbidly obese or those obese with coexisting conditions $^{(11)}$. Behavioural, non-pharmacological interventions promoting dietary restrictions show some promise in producing moderate, short-term weight loss and are associated with fewer adverse events than pharmacological or surgical interventions ${ }^{(13)}$. However, most studies have evaluated behavioural interventions in selected patient groups or in community groups, with few specifically targeting primary-care patients.

$\mathrm{UK}^{(14)}$ and Australian ${ }^{(15)}$ preventive guidelines recommend that primary-care physicians assess patients for overweight and obesity and develop appropriate weightmanagement plans. The US Preventive Services Task Force recommends that intensive counselling and behavioural interventions' be offered to all obese primary-care patients; with high intensity being defined as more frequent than monthly contact offered in the first 3 months of treatment ${ }^{(16)}$. A review by Tsai et al., which included studies conducted only in the USA, reported that the use of pharmacological treatment (i.e. sibutramine and orlistat) accompanied by brief physician counselling or the use of meal replacements with dietitian-delivered counselling were potentially effective strategies for weight reduction in primary-care patients ${ }^{(17)}$. As their review was limited just to studies conducted in the USA, there is a need to examine weight-loss interventions in other countries so that findings are relevant to practitioners located outside the US health-care system. With the recent removal of sibutramine from the European, US and Australian markets, findings regarding the effectiveness of this drug may no longer be relevant to practitioners. Further, consideration of the methodological rigour of studies is important to ensure that valid conclusions are drawn. The present review aims to describe the number, methodological rigour and effectiveness of behavioural intervention studies involving primarycare physicians that targeted weight loss in overweight or obese adult primary-care patients, met the Cochrane Effective Practice and Organisation of Care Group (EPOC) study design criteria $^{(18)}$ and were published between 1999 and 2011.

\section{Methods}

The MEDLINE, EMBASE, Cochrane, PsycINFO and SCOPUS databases were searched using the following search terms: 'obesity OR overweight OR weight loss' AND 'primary health care OR family practice OR general practice OR general practitioner OR physician patient relations OR guideline adherence'. The search was limited to completed studies, published in English from 1999 until December 2011. This time frame was selected because Tsai et al.'s review examining interventions in primary-care patients identified few studies published before 1999. The reference lists of relevant systematic reviews and studies were manually searched to identify additional studies. No additional studies were identified.

\section{Inclusion criteria}

\section{Participants}

Adult primary-care patients (aged $\geq 18$ years) who were overweight or obese (defined as $\mathrm{BMI} \geq 25 \cdot 0 \mathrm{~kg} / \mathrm{m}^{2}$ ) were included. Studies of interventions targeting specific patient groups (i.e. diabetes, hypertension) were included if the study specified overweight or obesity as an inclusion criteria.

\section{Interventions}

Studies aimed at reducing weight in overweight and obese primary-care patients were included. This encompassed behavioural interventions delivered by primary-care physicians alone or in conjunction with other personnel. Comparative trials where another intervention was compared with intervention(s) delivered by primary-care physicians were also included. Surgical and pharmacological interventions as well as studies where primary-care physicians were not involved in any component of the intervention were excluded.

\section{Outcomes}

Eligible studies included weight loss or/and reduction in BMI as an outcome. Weight/BMI change were chosen as the main outcomes as studies focused on other outcomes (such as physical activity levels, nutrition changes, biochemistry data) may not provide an adequate basis for identifying effective approaches for directly addressing overweight and obesity.

\section{Study design}

The following study designs that met the EPOC research criteria were included: randomised controlled trial (RCT), controlled clinical trial (CCT), controlled before-and-after study (CBA) and interrupted time series (ITS) ${ }^{(18)}$.

\section{Quality assessment}

The EPOC quality criteria for RCT, CCT and CBA were used to assess the methodological adequacy of 
included studies ${ }^{(19)}$. For each criterion a score of 'yes' was assigned if the study met the criterion, 'no' if it did not and 'unclear' if there was insufficient information to adequately decide if the criterion was met. A score out of nine for each study was reported.

\section{Data extraction}

The following were extracted by two authors independently.

\section{Participants and intervention}

Participant characteristics (including percentage of females, age, ethnicity and mean BMI) were extracted. Information related to the intervention, number of participants in each group, retention rate, mean weight change and whether statistically significant weight loss was achieved was also extracted. Whether a larger percentage of participants in the intervention group achieved clinically significant weight loss (for the purpose of the present review, this was defined as having a weight loss of more than $5 \%$ of initial body weight) compared with the control group was recorded. A weight loss of $5 \%$ or more of initial body weight has been shown to result in improvements in weight-related comorbidities ${ }^{(20,21)}$. Where two intervention arms existed, comparisons between intervention and control group were reported.

\section{Intensity}

Intensity of interventions were coded as 'low', 'moderate' or 'high' based on frequency of contact in the first 3 months. An intervention was defined as high intensity if there was more than monthly contact, moderate if monthly contact and low if less than monthly contact occurred in the first 3 months of the intervention ${ }^{(16)}$. Where there was insufficient information, intensity was coded as 'unsure'.

\section{Quality assurance}

All abstracts were reviewed by one researcher (S.L.Y.) and full-text articles of potentially relevant articles were retrieved. As a quality assurance measure, $10 \%$ of the abstracts were reviewed and coded independently by a second reviewer (M.C., A.G.). All coding for quality criteria and data extraction were carried out by two authors (S.L.Y., A.G.) and differences resolved by mutual discussion.

\section{Results}

A total of 1356 articles were obtained from the electronic search: Medline ( $n$ 933), Cochrane ( $n$ 105), SCOPUS ( $n$ 280) and PsycINFO ( $n$ 38).

Seventeen articles describing sixteen studies met the inclusion criteria (see Fig. 1). Martin et al. published findings from the same study at the end of the intervention $^{(22)}$ and 2 years' follow-up ${ }^{(23)}$. All included studies were RCT except for one, which was a $\mathrm{CBA}^{(24)}$. One study was included as an RCT, although only two out of the three study arms were randomised ${ }^{(25)}$. Only findings from the randomised groups were reported. Two studies did not have a control group but compared different interventions $^{(26,27)}$. A study by Wadden et al. was included although it had an intervention arm that included the use of pharmacology (sibutramine). Only results from the brief intervention group, which did not involve medication, are reported here ${ }^{(28)}$.

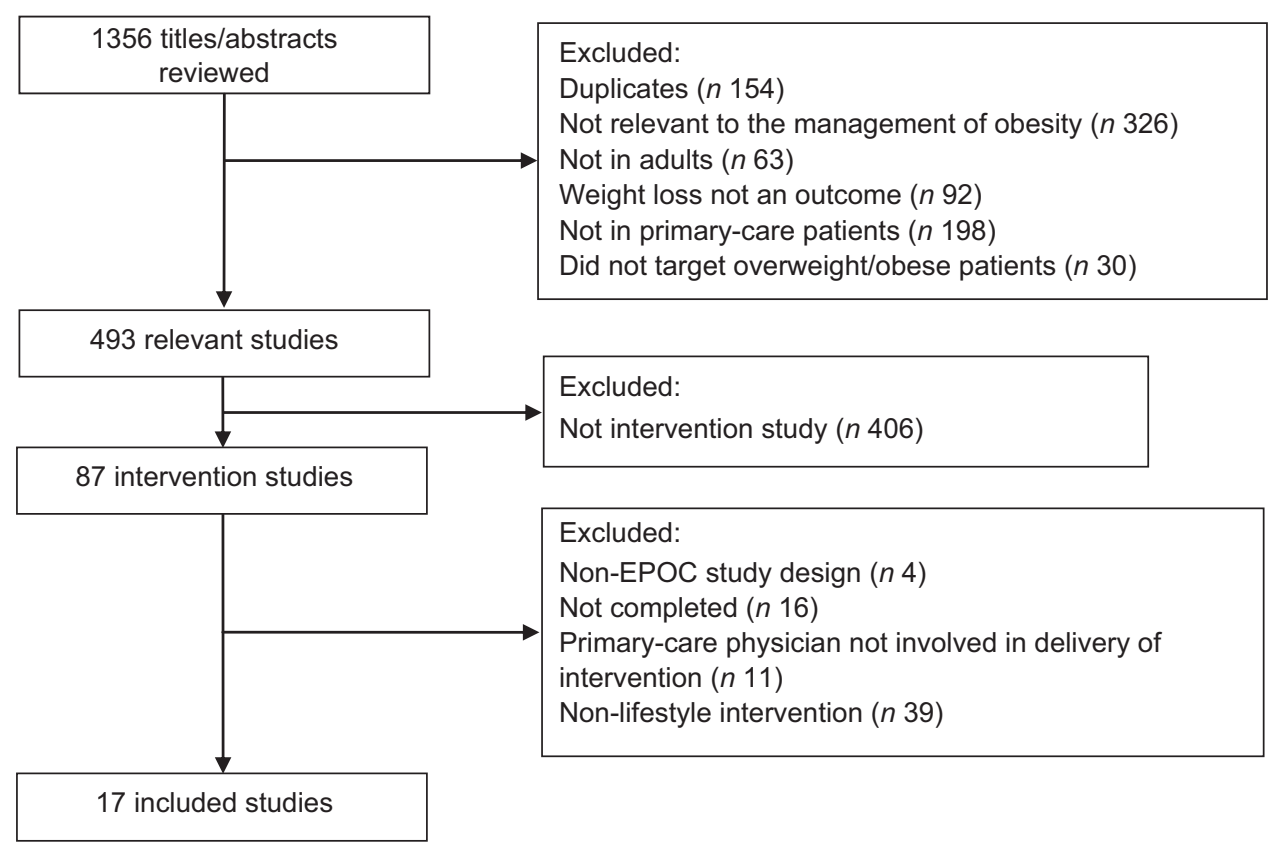

Fig. 1 Selection of articles for inclusion in the present systematic review (EPOC, Effective Practice and Organisation of Care Group) 
One study met all nine EPOC criteria ${ }^{(29)}$ and six others met seven or eight of the nine criteria ${ }^{(22,28,30-33)}$. Five studies met less than half the criteria ${ }^{(24-26,34,35)}$ (see Table 1). Adequate protection against contamination and study free from selective outcome reporting were the least likely criteria to have been sufficiently met. There was often inadequate information to determine whether mechanisms had been put in place to prevent contamination between the intervention and control groups. Only four studies had published protocols, thus allowing for assessment of whether selective outcome reporting had occurred ${ }^{(28-30,32)}$.

The interventions were broadly categorised into: (i) lifestyle counselling delivered primarily by primary-care physicians; (ii) lifestyle counselling delivered primarily by non-primary-care physicians; and (iii) multi-component intervention.

Six studies examined the effectiveness of lifestyle counselling delivered primarily by primary-care physicians (see Table 2). Of these, three tested the use of brief, tailored lifestyle counselling targeting dietary and/or exercise behaviour in changing patients' weight compared with usual care $^{(22,23,31,34)}$ and one examined the effect of a physiciandelivered group weight-management programme ${ }^{(25)}$. Two studies targeted providers, with one assessing the effectiveness of providing an educational intervention ${ }^{(29)}$ and the other testing the use of a sticker in overweight/obese patients' charts representing diagnosis and treatment or referral for the condition ${ }^{(24)}$. Of the six studies, three reported on low-intensity, one on moderate-intensity and two on high-intensity interventions.

None of the interventions targeting providers' behaviour resulted in statistically significant weight loss in their patients. Three studies targeting patients ${ }^{(22,23,25,34)}$ reported a statistically significant difference in amount of weight loss between the intervention and control group at end of intervention, with Martin et al. reporting significant weight loss at 6 months ${ }^{(22)}$, but no significant weight loss at 9 or 12 months $^{(23)}$. None of the studies reported that clinically significant weight loss was achieved.

Nine studies reported on the effectiveness of lifestyle counselling delivered by non-primary-care physicians, with support from primary-care physicians (see Table 3). The personnel delivering the intervention were allied health-care providers (including nurses or dietitians) or non-health-care providers. The types of interventions included meal replacements ${ }^{(26)}$, nurse- or dietitian-delivered counselling $^{(27,36,37)}$, weight-loss websites ${ }^{(30,32)}$ and counselling delivered by non-medical health coaches ${ }^{(28,32,33,38)}$. Two studies compared two interventions, without a control group $^{(26,27)}$. Four of the studies were high intensity, three were moderate intensity and one was low intensity. The number of sessions delivered during the first 3 months of the intervention was unclear in one study ${ }^{(27)}$, thus intensity could not be determined.

One of the two comparative effectiveness studies reported statistically significant findings. Ashley et al. found

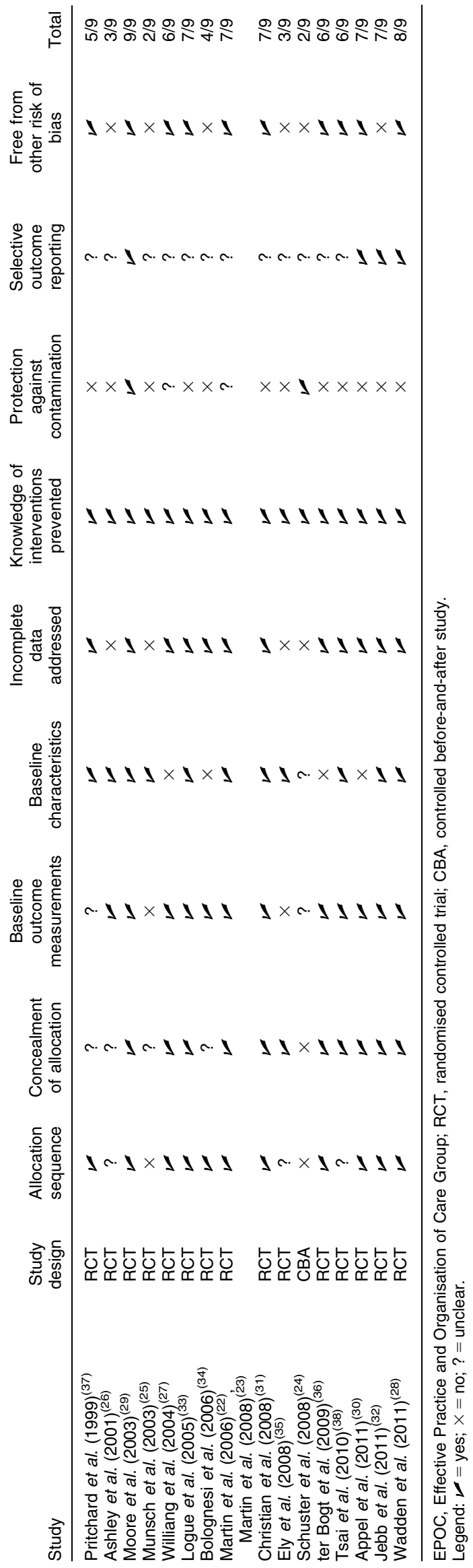


Table 2 Weight-loss interventions in primary-care patients delivered by primary-care physicians

\begin{tabular}{|c|c|c|c|c|c|c|c|c|c|c|c|}
\hline \multirow[b]{2}{*}{ Study, country } & \multicolumn{5}{|c|}{ Participants } & \multicolumn{3}{|l|}{ Intervention } & \multicolumn{3}{|c|}{ Results } \\
\hline & Comorbidities & $n$ & Sex (\%F) & Age (years) & $\mathrm{BMI}\left(\mathrm{kg} / \mathrm{m}^{2}\right)$ & Intervention details & Intensity & $\begin{array}{l}\text { Intervention } \\
\text { length }\end{array}$ & Retention rate & Summary & $\begin{array}{c}\text { Clinical } \\
\text { significance } \\
\text { (Y/N/DNR) }\end{array}$ \\
\hline $\begin{array}{l}\text { Moore et al. } \\
(2003)^{(29)} \text {, UK }\end{array}$ & n.s. & 843 & $\begin{array}{l}\text { IG: } 75 \% \\
\text { CG: } 73 \%\end{array}$ & $\begin{array}{l}\text { IG: } 48.4(\text { (sD 10.9) } \\
\text { CG: } 48 \cdot 8(\mathrm{sD} 12 \cdot 2)\end{array}$ & $\begin{array}{l}\text { IG: } 37 \cdot 0(5 \cdot 7) \\
\text { CG: } 36 \cdot 9(5 \cdot 8)\end{array}$ & $\begin{array}{l}\text { IG ( } n \text { 415): Three 90-min sessions } \\
\text { were delivered by dietitians to } \\
\text { general practitioners and practice } \\
\text { nurses. Training included clinical } \\
\text { benefits of weight loss and } \\
\text { effective treatments including } \\
\text { reduction of energy intake, } \\
\text { increased physical activity and } \\
\text { pharmaceutical interventions. } \\
\text { Practitioners were encouraged to } \\
\text { see patients biweekly until they } \\
\text { had lost } 10 \% \text { of their original } \\
\text { weight and then less frequently for } \\
\text { maintenance. At the end, } \\
\text { practices devised individualised } \\
\text { weight management protocols } \\
\text { based on this model } \\
\text { CG }(n \text { 428): Practices were asked to } \\
\text { provide usual care to their patients }\end{array}$ & High & 12 months & $\begin{array}{l}\text { At } 12 \text { months: } \\
67 \% \text { in IG; } \\
67 \% \text { in GC } \\
\text { At } 18 \text { months: } \\
62 \% \text { in IG; } \\
64 \% \text { in GC }\end{array}$ & $\begin{array}{l}\text { Difference }(\mathrm{IG}- \\
\mathrm{CG}): \uparrow 0 \cdot 6(95 \% \\
\mathrm{Cl}-2 \cdot 1,3 \cdot 2) \mathrm{kg} \\
\text { at } 3 \text { months; } \uparrow 1 \cdot 0 \\
(95 \% \mathrm{Cl}-1 \cdot 9, \\
3 \cdot 9) \mathrm{kg} \text { at } \\
12 \text { months; } \uparrow 1 \cdot 3 \\
(95 \% \mathrm{Cl}-1 \cdot 8, \\
4 \cdot 4) \mathrm{kg} \text { at } \\
18 \text { months }\end{array}$ & $\mathrm{N}$ \\
\hline $\begin{array}{l}\text { Munsch et al. } \\
(2003)^{(25)} \\
\text { Switzerland }\end{array}$ & n.s. & 122 & $\begin{array}{l}\text { IG: } 79 \cdot 2 \% \\
\text { CG: } 58 \cdot 8 \%\end{array}$ & $\begin{array}{l}\text { IG: females } 49 \\
\text { (SD 12); males } \\
45 \text { (SD 14) } \\
\text { CG: females } 49 \\
\text { (SD 10); males } \\
49 \text { (SD 10) }\end{array}$ & $\begin{array}{l}\text { IG: females } 35 \cdot 7 \\
\text { (SD 5.6); males } \\
36 \cdot 8 \text { (sD } 5 \cdot 2 \text { ) } \\
\text { CG: females } 34 \\
\text { (SD } 3.0 \text { ); males } \\
33.4 \text { (SD 2.5) }\end{array}$ & $\begin{array}{l}\text { IG }(n \text { 53)t: } 16 \text { group treatment } \\
\text { sessions focused on nutrition and } \\
\text { lifestyle (BASEL) were delivered } \\
\text { by physicians. Sessions were } \\
\text { delivered according to } \\
\text { standardised manual procedures } \\
\text { and covered nutrition, eating } \\
\text { behaviour, physical activity, social } \\
\text { competence and body image } \\
\text { CG }(n \text { 17)t: Patients received non- } \\
\text { specific comments about general } \\
\text { measures to lose weight }\end{array}$ & High & n.s. & $\begin{array}{l}\text { End of treatment: } \\
77 \% \text { in IG; } \\
71 \% \text { in CG } \\
\text { Between end of } \\
\text { treatment and } \\
1 \text {-year follow- } \\
\text { up: } 100 \% \text { in } \\
\text { IG; } 67 \% \text { in CG }\end{array}$ & $\begin{array}{l}\text { IG: } \downarrow 3 \cdot 8 \mathrm{~kg} \text { at } \\
\text { end of session; } \\
\downarrow 4 \cdot 7 \mathrm{~kg}^{\star \star \star} \text { at } \\
1 \text { year } \\
\text { CG: } \downarrow 0 \cdot 7 \mathrm{~kg} \text { at end } \\
\text { of session; } \\
\downarrow 0.4 \mathrm{~kg} \text { at } 1 \text { year }\end{array}$ & $\mathrm{N}$ \\
\hline $\begin{array}{l}\text { Bolognesi et al. } \\
(2006)^{(34)} \\
\text { Italy }\end{array}$ & n.s & 110 & $\begin{array}{l}\text { IG: } 43 \cdot 8 \% \\
\text { CG: } 62.5 \%\end{array}$ & $\begin{array}{c}\text { Overall: } 54 \cdot 2 \% \\
\text { aged }<50 \\
\text { IG: } 49 \cdot 2 \% \\
\text { aged }<50 \\
\text { CG: } 58 \cdot 3 \% \\
\text { aged }<50\end{array}$ & DNR & $\begin{array}{l}\text { IG }(n 55) \text { : The PACE protocol is a } \\
\text { method of physical activity } \\
\text { counselling tailored to participants' } \\
\text { stages of change. Patients } \\
\text { completed a PACE assessment } \\
\text { form and received counselling by } \\
\text { their GP (about } 2-5 \text { min). Patients } \\
\text { were asked to create a plan for } \\
\text { physical activity. A 2- to } 3 \text {-week } \\
\text { follow-up was conducted by phone } \\
\text { or mail } \\
\text { CG }(n \text { 55): Usual care was provided }\end{array}$ & Low & $2-3$ weeks & $\begin{array}{l}\text { At 5-6 months: } \\
\text { IG } 87 \cdot 3 \% \\
\text { CG: } 87 \cdot 3 \%\end{array}$ & $\begin{array}{l}\text { At 5-6 months: } \\
\text { IG } \mathrm{males} \\
\downarrow 0.78 \mathrm{~kg} / \mathrm{m}^{2 \star *} \\
\text { females } \\
\downarrow 0 \cdot 45 \mathrm{~kg} / \mathrm{m}^{2 \star \star} \\
\text { CG males } \\
\uparrow 0.57 \mathrm{~kg} / \mathrm{m}^{2} ; \\
\text { females } \\
\uparrow 0.3 \mathrm{~kg} / \mathrm{m}^{2}\end{array}$ & DNR \\
\hline
\end{tabular}


Table 2 Continued

\begin{tabular}{|c|c|c|c|c|c|c|c|c|c|c|c|}
\hline \multirow[b]{2}{*}{ Study, country } & \multicolumn{5}{|c|}{ Participants } & \multicolumn{3}{|l|}{ Intervention } & \multicolumn{3}{|c|}{ Results } \\
\hline & Comorbidities & $n$ & Sex (\%F) & Age (years) & BMI $\left(\mathrm{kg} / \mathrm{m}^{2}\right)$ & Intervention details & Intensity & $\begin{array}{l}\text { Intervention } \\
\text { length }\end{array}$ & Retention rate & Summary & $\begin{array}{c}\text { Clinical } \\
\text { significance } \\
\text { (Y/N/DNR) }\end{array}$ \\
\hline $\begin{array}{l}\text { Martin et al. } \\
\quad(2006)^{(22)} \\
\text { Martin et al. } \\
(2008)^{(23)} \\
\text { USA }\end{array}$ & n.s. & 144 & $100 \%$ & $\begin{array}{l}\text { IG: } 40 \cdot 69 \\
\text { (SD } 12 \cdot 59) \\
\text { CG: } 42 \cdot 97 \\
\text { (SD } 11 \cdot 38)\end{array}$ & $\begin{array}{l}\text { IG: } 38 \cdot 09 \\
\text { (sD } 7 \cdot 52) \\
\text { CG: } 39 \cdot 59 \\
\text { (sD } 7 \cdot 72)\end{array}$ & $\begin{array}{l}\text { All physicians received } 2 \mathrm{~h} \text { of training } \\
\text { on general obesity treatment } \\
\text { based on the NHLBI clinical } \\
\text { guidelines on obesity. IG } \\
\text { physicians received an additional } \\
7 \mathrm{~h} \text { of training } \\
\text { IG }(n 71) \text { : Participants received six } \\
\text { monthly treatment visits (lasting } \\
\sim 15 \text { min). Physicians received a } \\
\text { protocol prior to visits and } \\
\text { participants received oral and } \\
\text { written recommendations from } \\
\text { their physician. } \\
\text { Recommendations were prepared } \\
\text { by multidisciplinary teams with } \\
\text { input by physicians. } \\
\text { Recommendations were tailored } \\
\text { to the cultural backgrounds and } \\
\text { SES of patients } \\
\text { CG }(n \text { 73): Usual care was provided }\end{array}$ & Moderate & $\begin{array}{l}6 \text { months, } \\
18 \text { months }\end{array}$ & $\begin{array}{l}\text { IG: } 67 \cdot 6 \%(48 / 71) \\
\text { at } 6 \text { months } \\
\text { CG: } 79 \cdot 5 \% \\
\text { (58/73) at } \\
6 \text { months } \\
\text { IG+CG: } 44 \% \\
\text { (63/144) at } \\
9 \text { months; } \\
38 \% \text { (54/144) } \\
\text { at } 12 \text { months; } \\
35 \%(51 / 144) \\
\text { at } 18 \text { months }\end{array}$ & 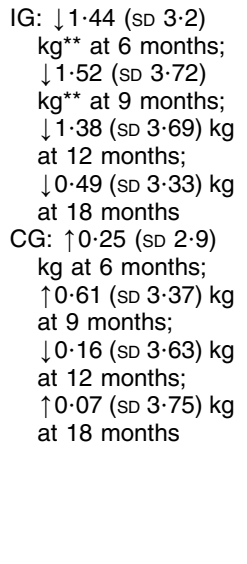 & $\mathrm{N}$ \\
\hline $\begin{array}{l}\text { Christian et al. } \\
(2008)^{(31)} \\
\text { USA }\end{array}$ & T2DM & 310 & $\begin{array}{l}\text { IG: } 65 \% \\
\text { CG: } 68 \%\end{array}$ & $\begin{array}{l}\text { IG: } 53 \cdot 0 \\
\text { (SD } 11 \cdot 25) \\
\text { CG: } 53 \cdot 4 \\
\text { (SD 10.70) }\end{array}$ & $\begin{array}{l}\text { IG: } 35 \cdot 4 \text { (sD 6.62) } \\
\text { CG: } 34 \cdot 8 \text { (sD } 7 \cdot 11)\end{array}$ & $\begin{array}{l}\text { IG ( } n \text { 155): Tailored feedback was } \\
\text { provided to patients (4-5 pages) } \\
\text { and their GP (brief summary) } \\
\text { based on a computer assessment } \\
\text { of patients' readiness to change } \\
\text { their physical activity and dietary } \\
\text { intake, and self-management } \\
\text { goals. Participants also received a } \\
\text { 30-page planning guide. GP } \\
\text { provided brief motivational } \\
\text { interviewing. Follow-up } \\
\text { consultations were held at } 3,6 \text { and } \\
9 \text { months post-baseline, and at } \\
\text { these visits GP reviewed patients' } \\
\text { progress with the goals they } \\
\text { had set } \\
\text { CG ( } n \text { 155): Participants received } \\
\text { a package of health education } \\
\text { materials }\end{array}$ & (1) & 12 months & $\begin{array}{l}\text { At } 12 \text { months: } \\
\text { IG } 91 \cdot 0 \% \\
\text { (141/155); } \\
\text { CG } 85 \cdot 2 \% \\
(132 / 155)\end{array}$ & $\begin{array}{l}\text { At } 12 \text { months: IG } \\
\downarrow 0 \cdot 18 \text { (sD } 10 \cdot 92) \\
\text { kg; CG } \uparrow 1.39 \\
\text { (SD } 10 \cdot 60) \mathrm{kg}\end{array}$ & $\mathrm{N}$ \\
\hline
\end{tabular}




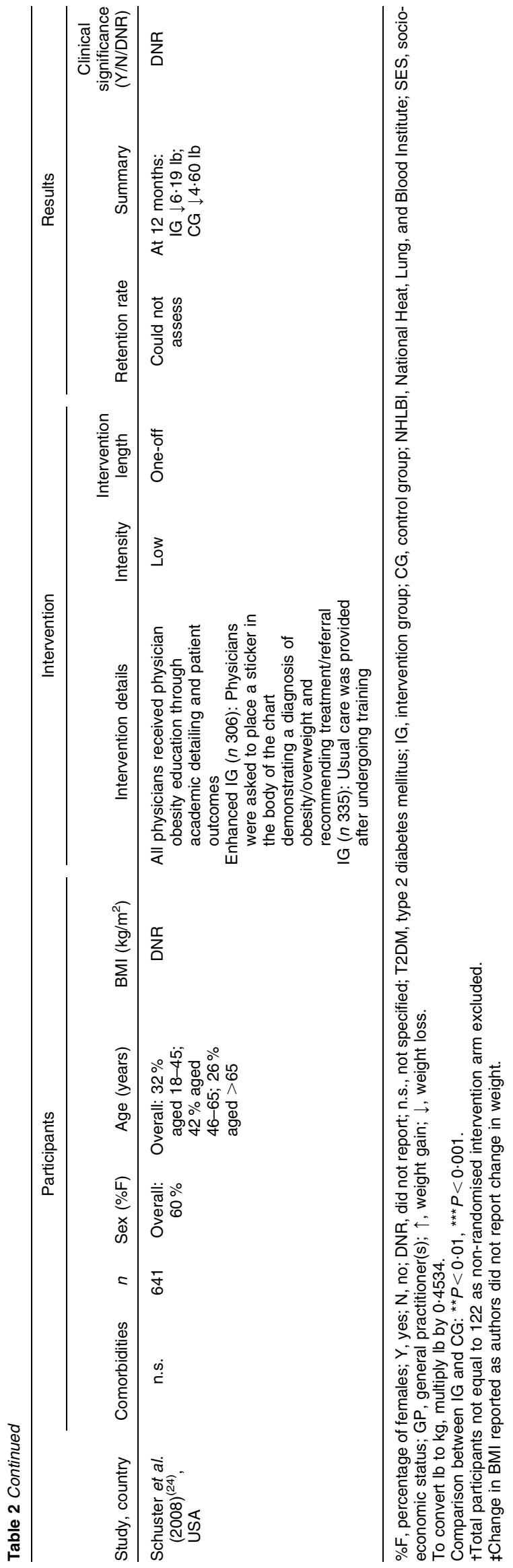

that the use of meal replacements in addition to lifestyle counselling by a dietitian produced greater weight loss than the other two interventions tested (dietitian counselling alone or counselling from primary-care physician and nurse practitioner plus meal replacements ${ }^{(26)}$. In Willaing et al.'s study, which compared nutrition counselling delivered by a dietitian with that delivered by a primary-care physician ${ }^{(27)}$, participants in both groups lost significantly more weight from baseline; however, there were no differences in weight loss between the two intervention groups. Six studies compared the intervention with either a usual care or minimal care group $^{(30,32,33,36-38)}$. Of them, four reported statistically significant results between usual care and intervention groups and that clinically significant weight loss was achieved $^{(30,32,37,38)}$. Three of the four effective studies involved weight-loss coaches delivering high-intensity behavioural counselling, with participants self-monitoring their dietary intake, physical activity and weight change.

Only one study ${ }^{(35)}$ examined a multi-component intervention involving a chronic care model (including electronic registry, decision support and patient selfmanagement support; see Table 4). The intervention was high intensity with a health counsellor who utilised motivational interviewing techniques. The study found that statistically significant weight loss was achieved compared with the usual care group; however, this was not clinically significant.

\section{Discussion}

The present review identified sixteen different intervention studies that met the specified inclusion criteria. The low number of studies identified is similar to a review conducted by Tsai and Wadden, where only ten studies targeting obesity in US primary-care settings were identified $^{(17)}$. In contrast to the review conducted by Tsai and Wadden, the current review included studies conducted outside the USA and excluded studies where pharmacological treatments were used. Due to the recent withdrawal of the weight-loss drug sibutramine from the market ${ }^{(39)}$, a number of studies included in Tsai and Wadden's review may no longer be relevant to practitioners. The removal of sibutramine in 2010 has resulted in orlistat being the only weight-loss medication available for practitioners located in Europe ${ }^{(39)}$. While other options exist for practitioners located in the USA, the overall limited availability and safety of weight-loss medications makes identifying effective behavioural interventions targeting excess weight an issue of critical importance. Given the high burden of illness associated with excess weight and the increasing discussion surrounding the use of primary care for weight management, the amount of research conducted is insufficient to inform practice. 
Table 3 Weight-loss interventions in primary-care patients delivered by non-primary-care physicians

\begin{tabular}{|c|c|c|c|c|c|c|c|c|c|c|c|}
\hline \multirow[b]{2}{*}{ Study, country } & \multicolumn{5}{|c|}{ Participants } & \multicolumn{3}{|c|}{ Intervention } & \multicolumn{3}{|c|}{ Results } \\
\hline & Comorbidities & $n$ & $\operatorname{Sex}(\% \mathrm{~F})$ & Age (years) & BMI $\left(\mathrm{kg} / \mathrm{m}^{2}\right)$ & Intervention details & Intensity & $\begin{array}{l}\text { Intervention } \\
\text { length }\end{array}$ & Retention rate & Summary & $\begin{array}{c}\text { Clinical } \\
\text { significance } \\
\text { (Y/N/DNR) }\end{array}$ \\
\hline $\begin{array}{l}\text { Pritchard et al. } \\
\text { (1999)(37), } \\
\text { Australia }\end{array}$ & HT, T2DM & 273 & $72.5 \%$ & $\begin{array}{l}73 \% \text { were } \\
\text { aged }<50\end{array}$ & DNR & $\begin{array}{l}\text { IG1 (dietitian only) ( } n \text { 89): } \\
\text { Dietitians invited patients to } \\
\text { join the study. Patients } \\
\text { received six counselling } \\
\text { sessions spaced equally } \\
\text { across } 12 \text { months. } \\
\text { Counselling focused on } \\
\text { principles of good nutrition and } \\
\text { exercise. Patients also kept } \\
\text { food records and diet history } \\
\text { IG2 (dieitian/GP) ( } n \text { 93): GP } \\
\text { invited patients to join study. } \\
\text { Patients saw the same GP on } \\
\text { two other occasions during the } \\
12 \text { months. The dietitian } \\
\text { coordinated the follow-up } \\
\text { appointments. Patients also } \\
\text { received counselling sessions } \\
\text { by a dietitian similar to IG1 } \\
\text { CG ( } n \text { 91): Patients received } \\
\text { results from the initial } \\
\text { screening and usual care from } \\
\text { their GP }\end{array}$ & Low & 12 months & $\begin{array}{l}\text { At } 12 \text { months, } \\
\text { overweight } \\
\text { group: IG1 } \\
55 \% \text { IG2 } \\
71 \% \text {; CG } 71 \%\end{array}$ & $\begin{array}{l}\text { At } 12 \text { months: IG1 } \\
\downarrow 5.6 \mathrm{~kg} ; \mathrm{IG} 2 \\
\downarrow 6.7 \mathrm{~kg}\end{array}$ & $\begin{array}{c}\mathrm{Y}(\mathrm{IG} 1,-6 \cdot 6 \% \\
\quad(95 \% \mathrm{Cl} 5 \cdot 8 \\
7 \cdot 6 \%) ; \mathrm{IG} 2 \\
-7.3 \% \\
(95 \% \mathrm{Cl} 5 \cdot 8 \\
7 \cdot 6 \%))\end{array}$ \\
\hline $\begin{array}{l}\text { Ashley et al. } \\
(2001)^{(26)} \text {, } \\
\text { USA }\end{array}$ & n.s. & 113 & $100 \%$ & $\begin{array}{l}\text { IG1: } 42 \cdot 3(\text { (sD } 4 \cdot 1) \\
\text { IG2: } 41 \cdot 0 \text { (sD } 4 \cdot 3) \\
\text { IG3: } 41 \cdot 0 \text { (sD } 5 \cdot 7)\end{array}$ & $\begin{array}{l}\text { IG1: } 29 \cdot 9 \text { (SD 2.6) } \\
\text { IG2: } 30 \cdot 1 \text { (sD 2.9) } \\
\text { IG3: } 30 \cdot 1 \text { (sD 3.7) }\end{array}$ & $\begin{array}{l}\text { IG1 ( } n \text { 23): Participants } \\
\text { attended } 26 \text { small group } \\
\text { classes consisting of } 8-10 \\
\text { people, each lasting } 1 \mathrm{~h} \text { and } \\
\text { delivered by an RD. Sessions } \\
\text { were conducted weekly for the } \\
\text { first } 3 \text { months, then biweekly } \\
\text { and monthly for the last } \\
6 \text { months. Dietary } \\
\text { recommendations included a } \\
\text { low-calorie diet with no more } \\
\text { than } 30 \% \text { energy from fat. } \\
\text { Participants completed } \\
\text { homework including self- } \\
\text { monitoring of food intake and } \\
\text { physical activity. In the second } \\
\text { year participants attended } \\
\text { monthly dietitian-led seminars } \\
\text { IG2 ( } n \text { 26): Same intervention } \\
\text { with RD as IG1 with meal } \\
\text { replacement for two main } \\
\text { meals } \\
\text { IG3 ( } n \text { 25): Patients met biweekly } \\
\text { with nurse (2/3) or physician } \\
\text { (1/3) for } 15 \text { min (26 sessions). } \\
\text { This group used meal } \\
\text { replacement for two } \\
\text { main meals }\end{array}$ & High & 24 months & $\begin{array}{l}\text { At } 12 \text { months: } \\
65.5 \% \\
\text { At } 24 \text { months: } \\
34.5 \%\end{array}$ & $\begin{array}{l}\text { IG1: } \downarrow 3.4 \text { (sD } 5 \cdot 4) \\
\mathrm{kg}^{*} \text { at } 12 \text { months; } \\
\downarrow 1 \cdot 6 \text { (sD } 4 \cdot 3) \mathrm{kg}^{*} \\
\text { at } 24 \text { months } \\
\text { IG2: } \downarrow 7 \cdot 7 \text { (sD } 7 \cdot 8) \mathrm{kg} \\
\text { at } 12 \text { months; } \\
\downarrow 7 \cdot 6 \text { (sD } 6 \cdot 8) \mathrm{kg} \text { at } \\
24 \text { months } \\
\text { IG3: } \downarrow 3.5 \text { (sD } 5 \cdot 5) \mathrm{kg} \\
\text { at } 12 \text { months; } \\
\downarrow 3.2 \text { (sD } 7 \cdot 1) \mathrm{kg} \text { at } \\
24 \text { months }\end{array}$ & $\begin{array}{l}Y \text { (IG2 v. IG1 } \\
\text { and IG3: } \\
P \leq 0.05)\end{array}$ \\
\hline
\end{tabular}


Table 3 Continued

\begin{tabular}{|c|c|c|c|c|c|c|c|c|c|c|c|}
\hline \multirow[b]{2}{*}{ Study, country } & \multicolumn{5}{|c|}{ Participants } & \multicolumn{3}{|c|}{ Intervention } & \multicolumn{3}{|c|}{ Results } \\
\hline & Comorbidities & $n$ & $\operatorname{Sex}(\% F)$ & Age (years) & $\mathrm{BMI}\left(\mathrm{kg} / \mathrm{m}^{2}\right)$ & Intervention details & Intensity & $\begin{array}{l}\text { Intervention } \\
\text { length }\end{array}$ & Retention rate & Summary & $\begin{array}{c}\text { Clinical } \\
\text { significance } \\
\text { (Y/N/DNR) }\end{array}$ \\
\hline $\begin{array}{l}\text { Logue et al. } \\
\quad(2005)^{(33)} \\
\text { USA }\end{array}$ & n.s. & 665 & $\begin{array}{l}\text { CG: } 67 \% \\
\text { IG: } 70 \%\end{array}$ & $\begin{array}{c}\text { CG: } 38 \% \text { aged } \\
\text { 40-49; } 42 \% \\
\text { aged } 50-59 ; \\
20 \% \text { aged } \\
60-69 \\
\text { IG: } 42 \% \text { aged } \\
40-49 ; 42 \% \\
\text { aged } 50-59 ; \\
16 \% \text { aged } \\
60-69\end{array}$ & $\begin{array}{l}\text { CG: } 22 \% \text { with } \\
\text { BMI } 25 \cdot 0-29 \cdot 9 ; \\
32 \% \text { with BMI } \\
30 \cdot 0-34 \cdot 5 ; \\
24 \% \text { with BMI } \\
35 \cdot 0-39 \cdot 0 ; \\
22 \% \text { with BMI } \\
40 \cdot 0+ \\
\text { IG: } 18 \% \text { with BMI } \\
25 \cdot 0-29 \cdot 9 ; \\
37 \% \text { with BMI } \\
30 \cdot 0-34 \cdot 5 ; \\
21 \% \text { with BMI } \\
35 \cdot 0-39 \cdot 0 ; \\
24 \% \text { with BMI } \\
40 \cdot 0+\end{array}$ & $\begin{array}{l}\text { CG ( } n \text { 336): Patients were asked } \\
\text { to provide anthropometric, } \\
\text { dietary and exercise } \\
\text { information every } 6 \text { months. } \\
\text { An RD provided } 10 \text { min of } \\
\text { counselling and prepared } \\
\text { prescriptions based on the } \\
\text { information provided } \\
\text { IG }(n \text { 329): Same as control plus } \\
\text { evaluation for anxiety, } \\
\text { depression and binge eating } \\
\text { disorder every } 6 \text { months and } \\
\text { SOC assessment. Patients } \\
\text { were mailed stage- and } \\
\text { behaviour-matched } \\
\text { workbooks that corresponded } \\
\text { to their SOC profile. Patients } \\
\text { also received brief monthly } \\
\text { telephone call from a weight- } \\
\text { loss advisor. Primary-care } \\
\text { physicians received periodic } \\
\text { reports summarizing patient } \\
\text { progress }\end{array}$ & Moderate & 24 months & $\begin{array}{c}\text { At } 24 \text { months: } \\
\text { CG } 79 \cdot 2 \% \text {; } \\
\text { IG } 82.4 \%\end{array}$ & $\begin{array}{l}\text { At } 24 \text { months: CG } \\
\downarrow 0.16 \text { (sD } 0.42) \\
\mathrm{kg} ; \mathrm{IG} \downarrow 0.39 \\
\text { (SD } 0.38) \mathrm{kg}\end{array}$ & $N$ \\
\hline $\begin{array}{l}\text { Willaing et al. } \\
(2008)^{(27)} \\
\text { Denmark }\end{array}$ & DysL, T2DM & 503 & $\begin{array}{l}\text { IG1: } 66 \% \\
\text { IG2: } 71 \%\end{array}$ & $\begin{array}{l}\text { IG1: } 54 \text { (range } \\
\text { 18-84) } \\
\text { IG2: } 50 \text { (range } \\
\text { 18-87) }\end{array}$ & $\begin{array}{c}\text { IG1: } 32 \cdot 5 \text { (range } \\
20 \cdot 7-51 \cdot 4 \text { ) } \\
\text { IG2: } 33 \cdot 7 \text { (range } \\
22 \cdot 0-58 \cdot 0 \text { ) }\end{array}$ & $\begin{array}{l}\text { IG1 (GP) ( } n \text { 191): GP received } \\
1 \mathrm{~d} \text { training in motivational } \\
\text { interviewing. Dietary } \\
\text { counselling delivered by GP } \\
\text { covered general advice and } \\
\text { delivery of commercially } \\
\text { available written information } \\
\text { on healthy eating. The initial } \\
\text { consultation with the GP was } \\
\text { approximately } 30 \text { min, with } \\
\text { subsequent consultations of } \\
12 \text { min } \\
\text { IG2 (dietitian) ( } n \text { 312): Dietary } \\
\text { counselling was delivered by a } \\
\text { dietitian; covering principles of } \\
\text { good nutrition, advice on food } \\
\text { shopping, meal planning, } \\
\text { cooking methods and } \\
\text { exercise. Reduction of energy } \\
\text { intake and fat were } \\
\text { recommended. The initial } \\
\text { session was } 1 \mathrm{~h} \text { with later } \\
\text { sessions of } 30 \text { min. GP were } \\
\text { provided with brief updates } \\
\text { every } 6 \text { months }\end{array}$ & Unclear & 12 months & $\begin{array}{l}\text { At } 12 \text { months: } \\
\text { IG1 } 68 \% ; \\
\text { IG2 } 67 \%\end{array}$ & $\begin{array}{l}\text { At } 12 \text { months: IG1 } \\
\downarrow 2 \cdot 5(95 \% \mathrm{Cl} \\
-3.74,-1 \cdot 26) \mathrm{kg} ; \\
\text { IG2 } \downarrow 3.2(95 \% \mathrm{Cl} \\
-3.87,-2 \cdot 53) \mathrm{kg}\end{array}$ & $\mathrm{N}$ \\
\hline
\end{tabular}


Table 3 Continued

\begin{tabular}{|c|c|c|c|c|c|c|c|c|c|c|c|}
\hline \multirow[b]{2}{*}{ Study, country } & \multicolumn{5}{|c|}{ Participants } & \multicolumn{3}{|c|}{ Intervention } & \multicolumn{3}{|c|}{ Results } \\
\hline & Comorbidities & $n$ & $\operatorname{Sex}(\% F)$ & Age (years) & BMI $\left(\mathrm{kg} / \mathrm{m}^{2}\right)$ & Intervention details & Intensity & $\begin{array}{l}\text { Intervention } \\
\text { length }\end{array}$ & Retention rate & Summary & $\begin{array}{c}\text { Clinical } \\
\text { significance } \\
\text { (Y/N/DNR) }\end{array}$ \\
\hline $\begin{array}{l}\text { ter Bogt et al. } \\
(2009)^{(36)} \\
\text { USA }\end{array}$ & HT, DysL & 457 & $\begin{array}{l}\text { IG: } 49 \cdot 8 \% \\
\text { CG: } 53 \cdot 9 \%\end{array}$ & $\begin{array}{l}\text { IG: } 55 \cdot 3 \text { (sD 7.7) } \\
\text { CG: } 56.9(\text { sD } 7 \cdot 8)\end{array}$ & $\begin{array}{l}\text { IG: } 29 \cdot 5 \text { (sD 3.1) } \\
\text { CG: } 29 \cdot 6 \text { (sD 3.6) }\end{array}$ & $\begin{array}{l}\text { All patients had to undergo } \\
\text { screening where weight, } \\
\text { height, metabolic } \\
\text { measurements were collected } \\
\text { and a lifestyle questionnaire } \\
\text { was administered } \\
\text { CG ( } n \text { 232): One visit } \\
\text { (approximately } 10 \text { min) with } \\
\text { GP to discuss result from } \\
\text { screening } \\
\text { IG }(n \text { 225): The NP had } 4 \mathrm{~h} \text { of } \\
\text { training using standardized } \\
\text { computer software that } \\
\text { contained instructions on } \\
\text { lifestyle counselling. Patients } \\
\text { received four individual visits } \\
\text { and one feedback session by } \\
\text { telephone by the NP in the first } \\
\text { year }\end{array}$ & Moderate & 12 months & $\begin{array}{l}\text { At } 12 \text { months: } \\
\text { IG } 89 \cdot 3 \% \\
(201 / 225) \text {; } \\
\text { CG: } 92 \cdot 7 \% \\
(215 / 232)\end{array}$ & 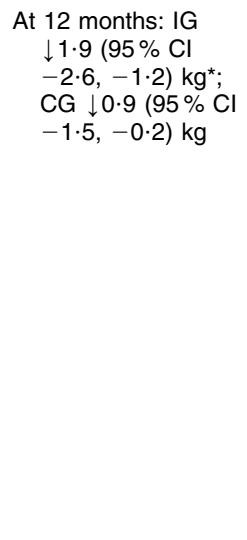 & $\mathrm{N}$ \\
\hline $\begin{array}{l}\text { Tsai et al. } \\
(2010)^{(38)} \text {, } \\
\text { USA }\end{array}$ & n.s. & 50 & DNR & $\begin{array}{l}\text { IG: } 51 \cdot 3 \text { (sD 2.3) } \\
\text { CG: } 47 \cdot 6(\text { SD } 2 \cdot 5)\end{array}$ & $\begin{array}{l}\text { IG: } 35 \cdot 4 \text { (SD 1.2) } \\
\text { CG: } 37.6 \text { (SD 1.1) }\end{array}$ & $\begin{array}{l}\text { CG }(n \text { 26): Patients met quarterly } \\
\text { with their PCP and were } \\
\text { provided with 1-2 page } \\
\text { handouts. Patients also } \\
\text { received a calorie counter, } \\
\text { pedometer and sample meal } \\
\text { plan. Each visit lasted } 2-3 \text { min } \\
\text { IG }(n 24) \text { : Same as control group } \\
\text { with additional eight brief visits } \\
\text { with an MA. Visits were } \\
\text { conducted using handouts } \\
\text { adapted by the DPP. Patients } \\
\text { were provided with strict } \\
\text { calorie consumption advice. } \\
\text { Patients also kept diaries of } \\
\text { food intake and advice to } \\
\text { increase physical activity }\end{array}$ & High & 12 months & $\begin{array}{l}\text { At } 6 \text { months: } \\
\text { IG } 87 \cdot 5 \% \text {; } \\
\text { CG } 92 \cdot 3 \% \\
\text { At } 12 \text { months: } \\
\text { IG } 96 \cdot 2 \% \text {; } \\
\text { CG } 91 \cdot 7 \%\end{array}$ & $\begin{array}{l}\text { IG: } \downarrow 4.4 \text { (sD } 0.6) \\
\mathrm{kg}^{\star \star \star} \text { at } 6 \text { months; } \\
\downarrow 2 \cdot 30 \text { (sD } 0.9) \mathrm{kg} \\
\text { at } 12 \text { months } \\
\text { CG: } \downarrow 0.9 \text { (sD } 0.6) \mathrm{kg} \\
\text { at } 6 \text { months; } \downarrow 1.1 \\
\text { (sD } 0.8 \text { ) kg) at } \\
12 \text { months }\end{array}$ & $\begin{array}{l}6 \text { months: } Y^{\star \star \star} \\
12 \text { months: } N\end{array}$ \\
\hline
\end{tabular}


Table 3 Continued

\begin{tabular}{|c|c|c|c|c|c|c|c|c|c|c|c|}
\hline \multirow[b]{2}{*}{ Study, country } & \multicolumn{5}{|c|}{ Participants } & \multicolumn{3}{|c|}{ Intervention } & \multicolumn{3}{|c|}{ Results } \\
\hline & Comorbidities & $n$ & $\operatorname{Sex}(\% F)$ & Age (years) & BMI $\left(\mathrm{kg} / \mathrm{m}^{2}\right)$ & Intervention details & Intensity & $\begin{array}{l}\text { Intervention } \\
\text { length }\end{array}$ & Retention rate & Summary & $\begin{array}{c}\text { Clinical } \\
\text { significance } \\
\text { (Y/N/DNR) }\end{array}$ \\
\hline $\begin{array}{l}\text { Appel et al. } \\
(2011)^{(30)} \\
\text { USA }\end{array}$ & $\mathrm{HT}, \mathrm{HC}, \mathrm{T} 2 \mathrm{DM}$ & 415 & $\begin{array}{l}\text { IG1: } 63 \cdot 3 \% \\
\text { IG2: } 63 \cdot 8 \% \\
\text { CG: } 63 \cdot 8 \%\end{array}$ & $\begin{array}{l}\text { IG1: } 55 \cdot 8 \text { (sD 9.7) } \\
\text { IG2: } 53 \cdot 3 \text { (sD 10.5) } \\
\text { CG: } 52 \cdot 9 \text { (sD 10.1) }\end{array}$ & $\begin{array}{l}\text { IG1: } 36 \cdot 0 \text { (SD } 4 \cdot 7) \\
\text { IG2: } 36.8(\text { (sD } 5 \cdot 12) \\
\text { CG: } 36.8(\text { SD } 5 \cdot 14)\end{array}$ & $\begin{array}{l}\text { IG1 ( } n \text { 139): Participants were } \\
\text { encouraged to log on weekly } \\
\text { to a website designed to help } \\
\text { with weight loss. Those who } \\
\text { had not logged on for } 7 \mathrm{~d} \\
\text { received an email reminder. } \\
\text { Weight-loss coaches } \\
\text { encouraged participants to } \\
\text { complete the modules on the } \\
\text { web page. } 12 \text { sessions by } \\
\text { phone were offered to } \\
\text { participants for the first } \\
3 \text { months. Participants received } \\
\text { one call a month for the } \\
\text { remainder of the intervention } \\
\text { IG2 }(n \text { 138): Participants were } \\
\text { encouraged to log on weekly } \\
\text { to the above website and } \\
\text { received reminder emails } \\
\text { similar to IG1. Participants } \\
\text { received nine in-person group } \\
\text { sessions and three individual } \\
\text { sessions for the first } 3 \text { months, } \\
\text { and three monthly contacts for } \\
\text { the rest of the intervention } \\
\text { CG }(n 138) \text { Participants met with } \\
\text { a weight-loss coach at } \\
\text { baseline and received } \\
\text { brochures and a list of } \\
\text { recommended websites } \\
\text { For the intervention groups, PCP } \\
\text { received a progress report on } \\
\text { their patients. Reminder } \\
\text { letters were sent on behalf of } \\
\text { PCP if participants were not } \\
\text { engaged in the study }\end{array}$ & High & 24 months & $\begin{array}{l}\text { At } 6 \text { months: } \\
88.2 \% \\
\text { At } 12 \text { months: } \\
85.5 \% \\
\text { At } 24 \text { months: } \\
94.5 \%\end{array}$ & 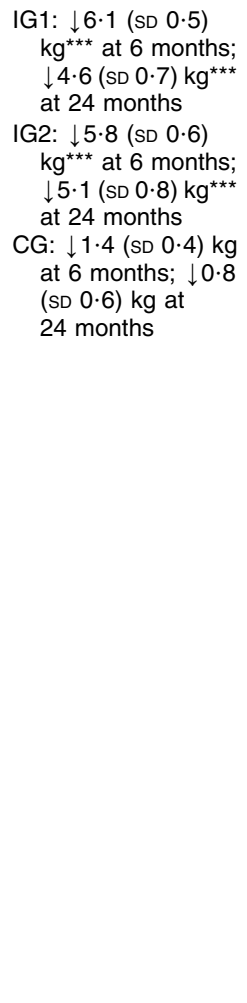 & $Y^{* \star *}$ \\
\hline
\end{tabular}




\begin{tabular}{|c|c|c|c|c|c|c|c|c|c|c|c|}
\hline \multirow[b]{2}{*}{ Study, country } & & & & & & & & & & & \\
\hline & Comorbidities & $n$ & $\operatorname{Sex}(\% F)$ & Age (years) & BMI $\left(\mathrm{kg} / \mathrm{m}^{2}\right)$ & Intervention details & Intensity & $\begin{array}{l}\text { Intervention } \\
\text { length }\end{array}$ & Retention rate & Summary & $\begin{array}{c}\text { Clinical } \\
\text { significance } \\
\text { (Y/N/DNR) }\end{array}$ \\
\hline $\begin{array}{l}\text { Jebb et al. } \\
(2011)^{(32)}, \\
\text { Australia, } \\
\text { Germany and } \\
\text { UK }\end{array}$ & $\begin{array}{l}\text { Central } \\
\text { adiposity, } \\
\text { T2DM without } \\
\text { insulin } \\
\text { treatment, } \\
\text { family history } \\
\text { of diabetes, } \\
\text { gestational } \\
\text { diabetes, } \\
\text { IGT, IFG, } \\
\text { DysL, HT, } \\
\text { PCOS, lower- } \\
\text { limb OA, } \\
\text { abdominal } \\
\text { hernia }\end{array}$ & 772 & $\begin{array}{l}\text { IG: } 88 \% \\
\text { CG: } 86 \%\end{array}$ & $\begin{array}{l}\text { IG: } 46 \cdot 5 \text { (sD 13.5) } \\
\text { CG: } 48 \cdot 2 \text { (sD 12.2) }\end{array}$ & $\begin{array}{l}\text { IG: } 31 \cdot 5 \text { (sD 2.6) } \\
\text { CG: } 31 \cdot 3(\text { (sD } 2 \cdot 6)\end{array}$ & $\begin{array}{l}\text { IG }(n \text { 377): Participants received } \\
\text { free access to weekly } \\
\text { community-based } \\
\text { WeightWatchers }{ }^{\circledR} \text { meetings. } \\
\text { Components of the } \\
\text { programme included } \\
\text { weigh-ins, group discussion, } \\
\text { behavioural counselling and } \\
\text { motivation, as well as Internet- } \\
\text { based system for monitoring } \\
\text { and peer support } \\
\text { CG }(n \text { 395): Participants } \\
\text { received weight loss advice } \\
\text { from a PCP using national } \\
\text { guidelines }\end{array}$ & High & 12 months & $\begin{array}{l}\text { End of } \\
\text { intervention } \\
\text { (12 months): } \\
\text { IG } 61 \cdot 0 \% \text {, } \\
\text { CG } 54 \cdot 2 \%\end{array}$ & $\begin{array}{l}\text { At } 12 \text { months: IG } \\
\downarrow 5.06 \text { (sD } 0.31) \\
\mathrm{kg}^{*} ; \mathrm{CG} \downarrow 2 \cdot 25 \\
\text { (SD } 0.21) \mathrm{kg}\end{array}$ & $\begin{array}{l}\text { Those in IG } \\
\text { had } \\
\text { increased } \\
\text { odds of } \\
\text { losing } 5 \% \text { or } \\
\text { more } \\
\text { (OR }=3 \cdot 0, \\
95 \% \mathrm{Cl} 2 \cdot 0 \text {, } \\
4 \cdot 4) \text { and } \\
10 \% \text { or more } \\
\text { (OR }=3 \cdot 2, \\
95 \% \mathrm{Cl} 2 \cdot 0 \text {, } \\
5 \cdot 3) \text { of their } \\
\text { initial body } \\
\text { weight }\end{array}$ \\
\hline $\begin{array}{l}\text { Wadden et al. } \\
(2011)^{(28)} \\
\text { USA }\end{array}$ & $\begin{array}{l}\text { At least two } \\
\text { of five } \\
\text { components } \\
\text { of the MetS }\end{array}$ & 390 & $\begin{array}{l}\text { IG: } 84 \cdot 0 \% \\
\text { CG: } 75 \cdot 4 \%\end{array}$ & $\begin{array}{l}\text { IG: } 52 \cdot 0 \text { (sD 12.2) } \\
\text { CG: } 51 \cdot 7(\text { (sD 12.1) }\end{array}$ & $\begin{array}{l}\text { IG } 38 \cdot 5(\text { SD } 4 \cdot 6) \\
\text { CG: } 39 \cdot 0(\text { (sD } 4 \cdot 8)\end{array}$ & $\begin{array}{l}\text { CG }(n \text { 130)t: Participants saw } \\
\text { their GP quarterly during the } \\
\text { study period. PCP provided } \\
\text { handouts and discussed this } \\
\text { information with patients, } \\
\text { using written protocols } \\
\text { provided by the research team } \\
\text { IG }(n \text { 131)t: Same as control plus } \\
10-15 \text { min monthly visit with } \\
\text { an MA, who delivered lifestyle } \\
\text { treatment based on the DPP. } \\
\text { In the first month, patients had } \\
\text { two visits with the MA. In the } \\
\text { second year, participants } \\
\text { could complete counselling } \\
\text { visits every other month by } \\
\text { phone, if they chose to }\end{array}$ & Moderate & 24 months & $\begin{array}{l}\text { At } 24 \text { months: IG } \\
85 \% \text {; CG } 85 \%\end{array}$ & $\begin{array}{l}\text { IG: } \downarrow 3 \cdot 4 \text { (sD } 0 \cdot 6) \mathrm{kg} \\
\text { at } 12 \text { months; } \\
\downarrow 2 \cdot 9 \text { (sD } 0 \cdot 7) \mathrm{kg}) \\
\text { at } 24 \text { months } \\
\text { CG: } \downarrow 2 \cdot 3(\mathrm{sD} 0 \cdot 6) \mathrm{kg} \\
\text { at } 12 \text { months; } \\
\downarrow 1 \cdot 7(\mathrm{sD} 0 \cdot 7) \mathrm{kg} \text { at } \\
24 \text { months }\end{array}$ & $\mathrm{N}$ \\
\hline
\end{tabular}

\%F, percentage of females; Y, yes; N, no; DNR, did not report; HT, hypertension; T2DM, type 2 diabetes mellitus; n.s., not specified; DysL, dyslipidaemia; HC, hypercholesterolaemia; IGT, impaired glucose tolerance IFG, impaired fasting glucose; PCOS, polycystic ovary syndrome; OA, osteoarthritis; MetS, metabolic syndrome; IG, intervention group; CG, control group; GP, general practitioner(s); RD, registered dietitian(s); SOC, stages of change; NP, nurse practitioner(s); PCP, primary-care physician(s); MA, medical assistant(s); DPP, Diabetes Prevention Program; $\uparrow$, weight gain; $\downarrow$, weight loss

†Total participants not equal to 390 as intervention arm which included use of pharmacology excluded. 
Table 4 Multi-component weight-loss intervention in primary-care patients

\begin{tabular}{|c|c|c|c|c|c|c|c|c|c|c|c|}
\hline \multirow[b]{2}{*}{ Study, country } & \multicolumn{5}{|c|}{ Participants } & \multicolumn{3}{|c|}{ Intervention } & \multicolumn{3}{|c|}{ Results } \\
\hline & Comorbidities & $\mathrm{n}$ & $\operatorname{Sex}(\% F)$ & Age (years) & BMI $\left(\mathrm{kg} / \mathrm{m}^{2}\right)$ & Intervention details & Intensity & $\begin{array}{l}\text { Intervention } \\
\text { length }\end{array}$ & $\begin{array}{l}\text { Retention } \\
\text { rate }\end{array}$ & Summary & $\begin{array}{c}\text { Clinical } \\
\text { significance } \\
\text { (Y/N/DNR) }\end{array}$ \\
\hline $\begin{array}{l}\text { Ely et al. } \\
(2008)^{(35)} \text {, } \\
\text { USA }\end{array}$ & n.s. & 107 & $\begin{array}{l}\text { IG: } 71 \% \\
\text { CG: } 83 \%\end{array}$ & $\begin{array}{l}\text { IG: } 49 \text { (SD 14) } \\
\text { CG: } 50 \text { (SD 15) }\end{array}$ & $\begin{array}{l}\text { IG: } 37 \text { (SD 8) } \\
\text { CG: } 36 \text { (SD 7) }\end{array}$ & $\begin{array}{l}\text { All physicians received } \\
\text { training and clinical } \\
\text { guidelines } \\
\text { IG ( } n \text { 51): Components } \\
\text { were derived using the } \\
\text { general principles of the } \\
\text { chronic care model. } \\
\text { This included: (i) clinical } \\
\text { information systems } \\
\text { consisting of an } \\
\text { electronic registry of } \\
\text { patients with regular } \\
\text { updates provided to } \\
\text { physicians and obesity } \\
\text { care recommendations; } \\
\text { (ii) decision support to } \\
\text { physicians via the } \\
\text { electronic registry; and } \\
\text { (iii) self-management } \\
\text { support for patients. } \\
\text { Patients also received } \\
\text { biweekly telephone- } \\
\text { based counselling from } \\
\text { counsellors for the first } \\
3 \text { months. Counselling } \\
\text { was structured using } \\
\text { motivational interviewing } \\
\text { CG ( } n \text { 56): Participants } \\
\text { received standard care }\end{array}$ & High & 3 months & $\begin{array}{l}\text { At } 90 \text { d: } 63 \% \\
\text { At } 180 \text { d: } 50 \%\end{array}$ & $\begin{array}{c}\text { IG: } \downarrow \downarrow 4 \cdot 5(\mathrm{sD} 7 \cdot 7) \\
\text { Ib at } 90 \mathrm{~d} ; \\
\downarrow 9 \cdot 4(\mathrm{sD} 10 \cdot 3) \\
\text { Ib** at } 180 \mathrm{~d} \\
\text { CG: } \downarrow 2 \cdot 4(\mathrm{sD} 8 \cdot 1) \\
\text { Ib at } 90 \mathrm{~d} ; \\
\downarrow 2 \cdot 1(\mathrm{sD} 10 \cdot 7) \\
\text { Ib at } 180 \mathrm{~d}\end{array}$ & DNR \\
\hline
\end{tabular}

$\% \mathrm{~F}$, percentage of females; Y, yes; N, no; DNR, did not report; n.s., not specified; IG, intervention group; CG, control group; $\downarrow$, weight loss.

To convert lb to kg, multiply lb by 0.4534 .

Comparison between IG and CG: ${ }^{* *} P<0 \cdot 01$. 
Overall, the studies were of moderate to good quality. One study met all EPOC quality criteria ${ }^{(40)}$. Two criteria which were poorly met across studies were selective outcome reporting and adequately protecting against contamination.

Only four studies included in the present review had published a study protocol ${ }^{(28-30,32)}$. Selectively reporting positive or statistically significant findings can lead to overestimation of treatment effects, subsequently affecting conclusions drawn from systematic reviews and meta analyses $^{(41)}$. Dwan et al. reported that discrepancies between protocol or trial registries and publications occur in a large proportion of studies, where at least one primary outcome was changed, introduced or omitted in $4-50 \%$ of trial reports ${ }^{(41)}$. Where a protocol does not exist, it is unknown whether selective outcome reporting occurred. Therefore, for a large number of studies included in the present review, the criterion related to selective outcome assessment could not be adequately assessed.

All studies except two ${ }^{(24,29)}$ used patients or physicians within the same practices as the unit of randomisation, thus increasing the likelihood of contamination between experimental and control groups. Contamination may reduce the effect size of the intervention due to the unintentional provision of additional care to control groups ${ }^{(42)}$. In order to improve the validity of findings, strategies need to be in place to ensure that the control group is not exposed to components of the intervention.

Selective outcome reporting and potential contamination may have affected findings from the included studies. Furthermore, poor reporting of study methodology in some studies made it difficult to assess study quality. These methodological and reporting shortcomings have been similarly reported in other reviews on weight $\operatorname{loss}^{(12,16,17,43)}$.

Of studies examining lifestyle counselling delivered by primary-care physicians, interventions that produced statistically significant weight loss included the use of a structured and tailored protocol to assist physicians with delivery of weight-loss counselling ${ }^{(22,23,25,34)}$. Consistent with current evidence ${ }^{(16)}$, regular contact between patients and physicians was a key component in producing weight loss, with higher-intensity interventions reporting larger amounts of weight loss. This contact may not need to be one on one; one study reported that group counselling sessions were effective in producing significant weight $\operatorname{loss}^{(25)}$. While one of the effective interventions ${ }^{(34)}$ was low intensity (one-off contact with physician), the amount of BMI change reported at 5-6 months' follow-up was marginal. The authors reported that highly motivated patients were enrolled in the intervention group with a large proportion of patients being in the contemplation and preparation stages of change, and may not have been reflective of usual primary-care patients ${ }^{(34)}$.

The two studies targeting providers did not report achieving any significant weight loss in their patients. Of the two, one was a high-intensity intervention ${ }^{(29)}$.
Although classified as high intensity, the intervention relied on practitioners' delivery of the proposed weightloss model (this entailed that practitioners saw their patients about once every fortnight until they had lost $10 \%$ of their initial body weight). The authors noted that practitioners' adherence to the intervention protocol was low, thus intensity could not be accurately estimated. Provider-targeted interventions for weight loss have been discussed in detail in other reviews ${ }^{(43-45)}$.

While a structured protocol to assist practitioners with delivery of weight-loss counselling appeared effective in producing some weight loss in overweight or obese patients, none of the interventions reported achieving clinically significant weight loss, making it questionable whether physician-delivered interventions alone are worth implementing in primary care.

In studies where non-physicians delivered the intervention, lifestyle counselling was conducted by allied health-care providers (nurses, dietitians) or non-health-care providers (weight-loss counsellors, medical assistants).

Two studies included a web-based component in addition to intensity lifestyle counselling ${ }^{(30,32)}$. Of these two, one used the web-based component in combination with referral to a community-based weight-loss programme (WeightWatchers $\left.{ }^{\circledR}\right)^{(38)}$ and the other with in-person or telephone support from weight-loss coaches ${ }^{(32)}$. Both studies utilised similar high-intensity interventions, with regular contact with health coaches or group leaders and Internet-based systems to help with self-monitoring and provide peer support. For both studies, participants in the intervention group lost significantly more weight than the control group (mean weight loss of approximately $6 \cdot 0 \mathrm{~kg}$ ). Appel et al. reported no significant difference in amount of weight loss between face-to-face and telephone support, suggesting there is potential for telephone counselling to be delivered as part of weight-reduction programmes to minimise intervention $\operatorname{cost}^{(30)}$.

Findings from studies where non-health-care providers delivered weight-management counselling were mixed. Tsai et al.'s high-intensity intervention reported that significantly more weight loss was achieved in the intervention group compared with the control group ${ }^{(38)}$, whereas studies by Wadden et $a l^{(28)}$ and Logue et $a l^{(33)}$ reported no significant difference in amount of weight loss between the intervention and control groups. Notably, the latter study compared the intervention with an 'augmented usual care group', where participants in the control group met with a dietitian for 10 min biannually ${ }^{(33)}$. This could have affected the control group's behaviour, thus making it harder to demonstrate an intervention effect.

These findings tentatively suggest that high-intensity interventions delivered by non-health-care providers in adjunct to primary-care physician consult are effective in producing clinically significant weight loss.

In studies involving allied health-care providers, the way in which weight-loss counselling was conducted 
varied depending on the personnel delivering the intervention. Where the dietitian was involved, delivery of the intervention largely relied on the dietitian to provide individualised advice and weight-loss strategies ${ }^{(37)}$. In contrast, nurse practitioners used a structured software program to assist with delivery of weight-loss counselling ${ }^{(36)}$. Both Pritchard et al. ${ }^{(37)}$ and ter Bogt et al. ${ }^{(36)}$ reported significantly more weight loss in the intervention group than the control group; however, only the Pritchard study involving dietitian-delivered advice reported that clinically significant weight loss was achieved. Pritchard et al.'s study highlighted the advantage of physician involvement in addition to dietitian-delivered care in increasing retention rate and proportion attending all sessions of the intervention ${ }^{(37)}$.

Other studies confirmed the effectiveness of dietitiandelivered interventions. Ashley et al. compared three interventions and found that dietitian-delivered advice coupled with meal replacements was effective in producing clinically significant weight loss compared with either receiving dietitian advice alone or using meal replacements coupled with primary-care physician and nurse practitioner counselling ${ }^{(26)}$. Analysis was conducted only on participants who completed the intervention. Therefore, treatment effect may have been overestimated. Despite this limitation, the study suggests that the use of meal replacements in conjunction with dietitian advice is useful in producing significant weight loss. Willaing et al. found no difference in the effectiveness of dietary counselling delivered by a primary-care physician compared with dietary counselling delivered by a dietitian ${ }^{(27)}$. Both groups had significant weight loss from baseline at 12 months, despite the primary-care physician spending less time during consultations than the dietitian.

Regardless of level of intervention intensity, dietitiandelivered counselling was effective in producing weight loss ranging from 3 to $6 \mathrm{~kg}$. Dietitians receive specialist training in nutrition assessment and counselling for weight loss and may therefore be more equipped to provide weight-management advice.

Findings from these studies suggest that high-intensity interventions involving non-physicians, with primary-care physicians playing a supportive role of assessment and referral, may be more effective than advice delivered by primary-care physicians alone in producing significant weight loss in overweight and obese primary-care patients. Comparisons made here, however, are limited by differences in intensity of intervention, with most primary-care physician-delivered interventions being of low to moderate intensity and non-primary-care physician-delivered interventions being of moderate to high intensity. These differences are likely to reflect clinical practice as primary-care physicians often face the need to deal with more acute issues and have less time to spend on delivery of lifestyle advice. The involvement of dietitians, non-health professionals or commercial weight-loss programmes enables intensive targeted counselling specifically dealing with weight management to be delivered to patients.

One study examined the use of a multi-component intervention which included an electronic registry, decision support and motivational interviewing delivered via telephone by a Master's level weight-loss advisor ${ }^{(35)}$. That study reported no statistically significant weight loss between the intervention and control group. The small sample size ( $n$ 101), short follow-up length and high drop-out rate made it difficult for any conclusions to be drawn.

\section{Practice implications}

Findings reported here suggest that intensive interventions delivered by non-physician personnel in the primary-care setting are effective in achieving clinically significant weight loss. There is insufficient evidence to suggest that counselling delivered by primary-care physicians alone produces clinically significant reductions in weight. However, involvement of primary-care physicians appears to increase retention rates and uptake of interventions delivered by non-physicians ${ }^{(37)}$. Approaches where non-physician providers play a more intensive role in delivery of behavioural interventions, accompanied by regular monitoring from primary-care physicians, could be a promising strategy to reduce obesity in primary-care patients. Given this finding, a review focused on assessing interventions solely delivered by non-primary-care physicians should be conducted to further inform weight management in this setting. The use of web-based interventions and meal replacements in adjunct with behavioural counselling (delivered by trained nonhealth providers or commercial centre weight-loss staff) appears promising. Additionally, delivery of interventions by dietitians appears effective regardless of intensity. With only few methodologically rigorous studies conducted, more studies evaluating the effectiveness of these interventions are needed. Future studies should also attempt to evaluate the acceptability, preference and uptake of these strategies among overweight and obese primary-care patients.

\section{Limitations}

The search terms used may not have identified all relevant studies. However, given the number of records extracted and the small proportion of relevant articles, it is likely that the majority of relevant articles were identified. The chance of missing relevant studies was further reduced by hand searching reference lists of relevant articles. Studies that examined behavioural interventions delivered in conjunction with medication were not examined as it was beyond the scope of the review.

\section{Conclusions}

Overall, the few studies identified and heterogeneity of interventions utilised made it difficult for conclusions to 
be drawn regarding what interventions are most effective in producing weight loss in overweight or obese primarycare patients. Given the burden of excess weight on the population and the advantage of using primary care to target weight loss, there is a need for more research exploring the use of this setting for delivery of weight-loss interventions. Results suggest that counselling delivered by non-physicians (face to face or telephone) with support from primary-care physicians is effective in producing weight loss. More studies assessing the effectiveness of these types of interventions are needed to confirm this.

\section{Acknowledgements}

Sources of funding: This work was supported by a grant from the beyondblue and National Heart Foundation of Australia Strategic Research Partnership Grant (G 08S 4042). M.C. is supported by Hunter Medical Research Institute Post-Doctoral Fellowships. Conflicts of interest: The authors declare no conflict of interest. Authors' contribution: S.L.Y. was responsible for the overall design of the study, conducted the literature search, extracted relevant data, interpreted findings and drafted the initial version of the article. M.C. played a major role in design of the study, interpretation of findings and revision of the article. R.S.-F. designed the study and provided critical comments regarding the intellectual content of the article. A.G. assisted in extraction of relevant data and revision of the draft manuscript. Acknowledgements: The authors wish to thank Shiho Rose for her assistance with screening for relevant articles.

\section{References}

1. World Health Organization (2011) Obesity and Overweight: Fact Sheets. Geneva: WHO; available at http:// www.who.int/mediacentre/factsheets/fs311/en/

2. Pi-Sunyer FX (2002) The obesity epidemic: pathophysiology and consequences of obesity. Obesity (Silver Spring) 10, Suppl. 2, 97S-104S.

3. World Health Organization (2000) Obesity: Preventing and Managing the Global Epidemic. WHO Technical Report Series no. 894. Geneva: WHO.

4. Pi-Sunyer FX (1993) Short-term medical benefits and adverse effects of weight loss. Ann Intern Med 119, 722-726.

5. Britt H, Miller GC, Charles J et al. (2010) General Practice Activity in Australia 2009-10. General Practice Series no. 27, Catalogue no. GEP27. Canberra: AIHW.

6. Hippisley-Cox J, Fenty J \& Heaps M (2007) Trends in Consultation Rates in General Practice 1995-2006: Analysis of the QRESEARCH database. http://www.ic.nhs.uk/webfiles/ publications/gp/QRESEARCH\%20Consultation\%20Rates \%20Report\%20FINAL.pdf (accessed September 2012).

7. Tan D, Zwar NA, Dennis SM et al. (2006) Weight management in general practice: what do patients want? Med J Aust 185, 73-75.

8. Thuan JF \& Avignon A (2005) Obesity management: attitudes and practices of French general practitioners in a region of France. Int J Obes (Lond) 29, 1100-1106.
9. Ruelaz AR, Diefenbach P, Simon B et al. (2007) Perceived barriers to weight management in primary care - perspectives of patients and providers. J Gen Intern Med 22, 518-522.

10. Sciamanna CN, Tate DF, Lang W et al. (2000) Who reports receiving advice to lose weight? Results from a multistate survey. Arch Intern Med 160, 2334-2339.

11. Colquitt JL, Picot J, Loveman E et al. (2009) Surgery for obesity. Cochrane Database Syst Rev issue 2, CD003641.

12. Norris SL, Zhang X, Avenell A et al. (2004) Efficacy of pharmacotherapy for weight loss in adults with type 2 diabetes mellitus: a meta-analysis. Arch Intern Med 164, 1395-1404.

13. Norris SL, Zhang X, Avenell A et al. (2005) Long-term non-pharmacological weight loss interventions for adults with prediabetes. Cochrane Database Syst Rev issue 2, CD005270.

14. National Institute for Health and Clinical Excellence (2006) Obesity: guidance on the prevention, identification, assessment and management of overweight and obesity in adults and childen. NICE clinical guideline no. 43. http:// www.nice.org.uk/CG43 (accessed September 2012).

15. RACGP National Standing Committe-Quality Care (2006) Overweight and obesity: Policy endorsed by the 48th RACGP Council 26 July 2006. http://www.racgp.org.au/ policy/Obesity_policy.pdf (accessed September 2012).

16. McTigue KM, Harris R, Hemphill B et al. (2003) Screening and interventions for obesity in adults: summary of the evidence for the US Preventive Services Task Force. Ann Intern Med 139, 933-949.

17. Tsai AG \& Wadden TA (2009) Treatment of obesity in primary care practice in the United States: a systematic review. J Gen Intern Med 24, 1073-1079.

18. Cochrane Effective Practice and Organisation of Care Review Group (2008) Data collection checklist. http:// epoc.cochrane.org/sites/epoc.cochrane.org/files/uploads/ datacollectionchecklist.pdf (accessed September 2012).

19. Cochrane Effective Practice and Organisation of Care Review Group (2009) Risk of bias. http://epoc.cochrane.org/ sites/epoc.cochrane.org/files/uploads/Suggested\%20risk $\% 20$ of $\% 20$ bias $\% 20$ criteria $\% 20$ for $\% 20$ EPOC $\% 20$ reviews.pdf (accessed September 2011).

20. Wing RR, Lang W, Wadden TA et al. (2011) Benefits of modest weight loss in improving cardiovascular risk factors in overweight and obese individuals with type 2 diabetes. Diabetes Care 34, 1481-1486.

21. National Health and Medical Research Council (2003) Clinical Practice Guidelines for the Management of Overweight and Obesity in Adults. Canberra: Australian Government, Department of Health and Ageing.

22. Martin PD, Rhode PC, Dutton GR et al. (2006) A primary care weight management intervention for low-income AfricanAmerican women. Obesity (Silver Spring) 14, 1412-1420.

23. Martin PD, Dutton GR, Rhode PC et al. (2008) Weight loss maintenance following a primary care intervention for low-income minority women. Obesity (Silver Spring) 16, 2462-2467.

24. Schuster RJ, Tasosa J \& Terwoord NA (2008) Translational research - implementation of NHLBI obesity guidelines in a primary care community setting: the Physician Obesity Awareness Project. J Nutr Health Aging 12, issue 10, 764S-769S.

25. Munsch S, Biedert E \& Keller U (2003) Evaluation of a lifestyle change programme for the treatment of obesity in general practice. Swiss Med Wkly 133, 148-154.

26. Ashley JM, St Jeor ST, Schrage JP et al. (2001) Meal replacements in weight intervention. Arch Intern Med 161, 1599-1604.

27. Willaing I, Ladelund S, Jorgensen T et al. (2004) Nutritional counselling in primary health care: a randomized comparison of an intervention by general practitioner or dietician. Eur J Cardiovasc Prev Rehabil 11, 513-520. 
28. Wadden TA, Volger S, Sarwer DB et al. (2011) A two-year randomized trial of obesity treatment in primary care practice. N Engl J Med 365, 1969-1979.

29. Moore H, Summerbell CD, Greenwood DC et al. (2003) Improving management of obesity in primary care: cluster randomised trial. BMJ 327, 1085.

30. Appel LJ, Clark JM, Yeh H-C et al. (2011) Comparative effectiveness of weight-loss interventions in clinical practice. $N$ Engl J Med 365, 1959-1968.

31. Christian JG, Bessesen DH, Byers TE et al. (2008) Clinicbased support to help overweight patients with type 2 diabetes increase physical activity and lose weight. Arch Intern Med 168, 141-146.

32. Jebb SA, Ahern AL, Olson AD et al. (2011) Primary care referral to a commercial provider for weight loss treatment versus standard care: a randomised controlled trial. Lancet 378, 1485-1492.

33. Logue E, Sutton K, Jarjoura D et al. (2005) Transtheoretical model - chronic disease care for obesity in primary care: a randomized trial. Obes Res 13, 917-927.

34. Bolognesi M, Nigg CR, Massarini M et al. (2006) Reducing obesity indicators through brief physical activity counseling (PACE) in Italian primary care settings. Ann Behav Med 31, 179-185.

35. Ely AC, Banitt A, Befort C et al. (2008) Kansas primary care weighs in: a pilot randomized trial of a chronic care model program for obesity in 3 rural Kansas primary care practices. J Rural Health 24, 125-132.

36. ter Bogt NC, Bemelmans WJ, Beltman FW et al. (2009) Preventing weight gain: one-year results of a randomized lifestyle intervention. Am J Prev Med 37, 270-277.
37. Pritchard DA, Hyndman J \& Taba F (1999) Nutritional counselling in general practice: a cost effective analysis. I Epidemiol Community Health 53, 311-316.

38. Tsai AG, Wadden TA, Rogers MA et al. (2010) A primary care intervention for weight loss: results of a randomized controlled pilot study. Obesity (Silver Spring) 18, 1614-1618.

39. Sayburn A (2010) Withdrawal of sibutramine leaves European doctors with just one obesity drug. BMJ 340, c477.

40. Moore BJ (2003) Supersized America: help your patients regain control of their weight. Cleve Clin J Med 70, 237-240.

41. Dwan K, Altman DG, Cresswell L et al. (2011) Comparison of protocols and registry entries to published reports for randomised controlled trials. Cochrane Database Syst Rev issue 1, MR000031.

42. The Cochrane Collaboration (2011) Cochrane Handbook for Systematic Reviews of Interventions Version 5:1.0. http://www.cochrane-handbook.org/ (accessed September 2011).

43. Harvey EL, Glenny AM, Kirk SFL et al. (2002) An updated systematic review of interventions to improve health professionals' management of obesity. Obes Rev 3, 45-55.

44. Harvey EL, Glenny AM, Kirk SF et al. (1999) A systematic review of interventions to improve health professionals' management of obesity. Int J Obes Relat Metab Disord 23, 1213-1222.

45. Flodgren G, Deane K, Dickinson HO et al. (2010) Interventions to change the behaviour of health professionals and the organisation of care to promote weight reduction in overweight and obese people. Cochrane Database Syst Rev issue 3, CD000984. 